ISSN: 0212-0267

DOI: https://doi.org/I0.I420I/hedu202I405II519

\title{
DATOS BIOGRÁFICOS DE LOS AUTORES
}

\section{About the authors}

Thomas S. Popkewitz es catedrático en el Department of Curriculum and Instruction de la Universidad de Wisconsin-Madison. Sus estudios e investigaciones se refieren a la política cultural y la historia del conocimiento educativo en la educación, la formación del profesorado y las ciencias sociales y psicológicas. Su investigación actual se centra en la epistemología social y la razón comparada inscritas en las ciencias relacionadas con la educación. Sus libros más recientes son The Impracticality of Practical Research. A History Of Sciences Of Change That Conserve and Excludes (University of Michigan, 2020; versión española en Barcelona, Octaedro,202I) y The international emergence of educational sciences in the post-World War Two years: Quantification, visualization, and making kinds of people (Routledge, editado con D. Pettersson y K. Hsiao). orcid.org/oooo-0002-5058-2235. Correo-e: popkewitz@education.wisc.edu

Miguel Á. Pereyra-García Castro es catedrático emérito de Educación Comparada de la Universidad de Granada. Se formó como pedagogo e historiador en las Universidades de Valencia y Columbia de Nueva York. Ha sido presidente de la Comparative Education Society in Europe (CESE) y ha investigado y enseñado como catedrático invitado en la Universidad Humboldt de Berlín; en la Universidad de WisconsinMadison como catedrático Tinker, y en la Universidad de Indiana, en Indianápolis, como Senior Fulbright Scholar in Residence. Ha participado en diversos proyectos de investigación de la Comisión Europea y españoles y actualmente dirige el proyecto «Retos y desafíos de la implantación de la formación dual en la formación profesional española», financiado por el Ministerio de Investigación. Ha publicado numerosos artículos y capítulos de libros sobre historia social y cultural de la educación, reformas de la formación del profesorado y programas internacionales de evaluación como el PISA de la OCDE. En 2016 editó con Barry Franklin para Routledge Systems of Reason and the Politics of Schooling. School Reform and Sciences of Education in the Tradition of Thomas S. Popkewitz. https://orcid.org/oooo-0002-6860-0315-. Correo-e: mpereyra@ugr.es

Raimundo Cuesta Fernández. Doctor en Historia por la Universidad de Salamanca. Ha sido catedrático de Historia en el IES Fray Luis de León de esa misma ciudad. Colaborador con universidades españolas e iberoamericanas, es especialista en historia de las disciplinas escolares, historia de la educación, didáctica las ciencias sociales y las relaciones entre memoria e historia. Premio Nacional a la Innovación Educativa en 1985, cofundador del grupo Cronos y de Fedicaria. Pertenece al Consejo de Redacción de la revista Con-Ciencia Social. Especialista en la historia de las disciplinas escolares y en la relación entre genealogía, historia conceptual y memoria. Sus dos últimos libros son: Las lecciones de Tersites. Semblanza de una vida y una época (Madrid, Vision 
Libros, 20I7) y Verdades sospechosas. Religión, historia y capitalismo (Madrid, Vision Libros, 2019). Véase relación y acceso más completo a sus publicaciones en www.nebraskaria.org.Correo-e: raicuesta2@gmail.com

Jesús Romero Morante trabaja en la Facultad de Educación de la Universidad de Cantabria como profesor titular de Didáctica de las Ciencias Sociales. Miembro del grupo Asklepios y la Federación Icaria (Fedicaria), ha sido editor asociado, para lengua española, de la revista Education Policy Analysis Archives. Es autor de numerosas publicaciones sobre didáctica crítica de las ciencias sociales, educación para la ciudadanía, innovación didáctica, formación del profesorado e historia del currículum. Entre sus publicaciones relativas a esta última línea de trabajo cabe destacar, además de las citadas en el artículo, las siguientes: «Educación para la ciudadanía y currículum: sus regímenes de verdad en perspectiva histórica», en Borghi, B.; García, F. F. y Moreno, O. (eds.): Novi cives: cittadini dall'infanzia in poi, Bologna, Pàtron Editore, 2015, pp. 63-76; o «La historia del currículum como fuente para la Didáctica de las Ciencias Sociales», REIDICS, Revista de Investigación en Didáctica de las Ciencias Sociales, 3 (2018), pp. I03-II8. ORCID: https://orcid.org/oooo-ooo2-2889-6oI9. Correo-e: romeroj@unican.es

Marta EsTellés Frade es doctora en Educación y profesora ayudante de Didáctica de las Ciencias Sociales en el Departamento de Educación de la Universidad de Cantabria. Miembro de Fedicaria, forma parte del Consejo Editor de la revista Con-Ciencia Social (Segunda época). Su investigación se ha desarrollado en el campo de la educación para la ciudadanía y la formación inicial del profesorado. Sus aportaciones recientes más relevantes son: «Governmentality, Curriculum Policies, and Citizenship Education: The Need for Historical Research» (en coautoría), en Pineda, J. A.; Alba, N. de y Navarro, E. (eds.): Handbook of Research on Education for Participative Citizenship and Global Prosperity, Hershey, IGI Global, 2019; «Tacit Assumptions of Citizenship Education: A Case Study in Spanish Initial Teacher Education» (en coautoría), en Education, Citizenhip and Social Justice, en prensa; "Global Citizenship Education in Teacher Education: is There any Alternative beyond Redemptive Dreams and Nightmarish GERMS?» (en coautoría), en Wolhuter, C. y Schugurensky, D. (eds.): Citizenship Education in Teacher Education: Theoretical and Practical Issues, New York, Routledge, en prensa; «Las huellas de la socialización política y escolar en las actitudes hacia la educación para la ciudadanía de los futuros maestros» (en coautoría), en Puig, M.; Ferreras, M. y Moreno, O. (eds.): Innovación e investigación en la formación inicial del profesorado desde las Didácticas de las Ciencias Experimentales y Sociales, Barcelona, Octaedro, en prensa. ORCID: https://orcid.org/oooo-oooI-61623875. Correo-e: marta.estelles@unican.es

Antti SAARI es profesor titular de la Facultad de Educación y Cultura de la Universidad de Tampere en Finlandia. Sus principales intereses de investigación son la historia y la filosofía de la educación y los estudios curriculares. Su investigación abarca dos temas principales: las interfaces entre los conocimientos de los expertos y el gobierno de la educación, incluido el modo en que los discursos psicológicos y sociológicos se traducen en prácticas de evaluación, gestión de las aulas y uso de la tecnología educativa. En particular, Saari ha estudiado los discursos psicológicos del bienestar subjetivo en forma de educación positiva y mindfulness, así como el papel de las teorías psicológicas 
y los conceptos de aprendizaje en el gobierno de la educación. ORCID: https://orcid. org/oooo-ooo3-3777-6948. Correo-e: antti.saari@tuni.fi

Tuomas Tervasmäki es investigador en la Facultad de Educación y Cultura de la Universidad de Tampere. Sus principales áreas de interés son la filosofía de la educación, los estudios curriculares y las cuestiones de inclusión y exclusión en la educación. En su investigación, que pronto será defendida como doctorado,, analiza la política de la educación y la política educativa finlandesa desde la perspectiva de la teoría del discurso político (también conocida como ideología y análisis del discurso). Actualmente, su foco es el funcionamiento de la ideología en las instituciones y prácticas educativas. ORCID: http://orcid.org/oooo-0002-2300-645. Correo-e: tuomas. tervasmaki@tuni.fi

Maja Plum es doctora en Educación por la Universidad de Copenhague. Lleva muchos años investigando la relación entre las formas de gobernar y la práctica educativa. Su libro El profesionalismo en una era de la documentación se publicó en danés en 2014. A nivel internacional ha publicado trabajos tanto antropológicos como históricos en revistas como Journal of Education policy; Ethnography and Education; Pedagogy, Culture and Society y Discourse - Studies in the Cultural Politics of Education. Viene desde hace años trabajando las obras de Michel Foucault concibiéndolas como lente analítico importante. Su artículo «Humanism and Administration» fue seleccionado por Stephen Ball para incluirlo en la edición de su libro Foucault and Education: Putting Theory to Work (2018). En 2019 dejó el mundo académico en la Universidad de Copenhague para trabajar como consultora para el Sindicato de Maestros de Jardín de Infancia de Dinamarca. ORCID: https://orcid.org/oooo-0002-3029-0593.Correo-e: mplum@hum.ku.dk

Melissa Andrade-Molina es profesora asociada en el Instituto de Matemáticas de la Pontificia Universidad Católica de Valparaíso en Chile. Ella tiene un PhD otorgado por la Facultad de Matemáticas y Física de la Universidad de Aalborg, Dinamarca. Sus principales intereses de investigación son las perspectivas sociopolíticas en educación. Particularmente, cómo, históricamente, el currículum escolar ha tenido (d) efectos de poder en la producción de subjetividades científicas, lo que ha denominado «the Mindniac». Su trabajo se ha dirigido a desafiar las narrativas dominantes en educación. Actualmente, ella está interesada en cómo la diversidad en las escuelas ha sido posicionada como una amenaza para el crecimiento económico, el nacimiento de la sala de clases -the class/room- y la importancia otorgada a las matemáticas escolares en la producción de ciudadanos para la sociedad. Correo-e: melissa.andrade@pucv.cl

Jennifer Díaz es profesora titular en la Universidad de Augsburgo en Minneapolis, MN. $\mathrm{Su}$ investigación se centra en los estudios curriculares, la reforma escolar y la formación del profesorado, especialmente en lo que respecta a la educación matemática elemental. Su reciente libro, The Paradox of Making In/Equality: A Cultural History of Reforming Math for All, analiza cómo las concepciones culturales de la identidad y la diferencia se producen en los planes de estudio y currículos de Estados Unidos, por lo que remodelan históricamente los términos de la inclusión y la exclusión. El alcance de sus investigaciones y estudios plantea cuestiones sobre los límites y las posibilidades de enseñar matemáticas de forma inclusiva y equitativa. ORCID: https://orcid.org/oooo-0003-3379-1593. Correo-e: diazj@augsburg.edu 
John P. Ivens obtuvo su PhD en Estudios del Currículum y Estudios Globales en el Departamento de Currículum y Enseñanza de la Universidad de Wisconsin-Madison. En la actualidad es assistant professor en «Estudios Sociales en Educación» del Glenville State College (en Glenville, Virginia Occidental). Viene investigando sobre la intersección de los estudios culturales y la historia de la cultura y la filosofía y sociología de las ciencias humanas a lo largo del siglo xx, junto a los estudios de Ciencia, Tecnología y Sociedad (STS) y la producción social del conocimiento. Todo ello, en el contexto histórico del nuevo mundo que emerge de la Segunda Guerra Mundial y la producción de un nuevo tipo de conocimiento en la vida cotidiana de las poblaciones, que conllevó también la producción de tesis culturales de cómo deberían ser los escolares. Ha publicado recensiones en relevantes revistas como History of Education y el estudio «One Kind of Human Being: MACOS, the Humans Ciencies, and Governmentality» (en European Education, en el número 3 del vol. 45, 20I4). ORCID: 0000-0003-0459-2I73. Correo-e: jpivens@wisc.edu

LeiZHeNG obtuvo su PhD en el Department of Curriculum E Instruction de la Universidad de Wisconsin-Madison, en donde hizo sus estudios de doctorado. Antes fue graduada en Historia en la Universidad de Nanjing para luego obtener un Master of Arts en estudios del currículum en la East China Normal University. Recientemente, la AERA (American Educational Research Association) le otorgó el Graduate Student Award en el SIC (Special Interest Group) sobre Critical Issues in Cultural Studies. La investigación de Zheng entrelaza los estudios de ciencia y tecnología (STS/CTS) y la geografía política con los estudios curriculares, con objeto sobre todo de analizar cómo se construyen históricamente diferentes infraestructuras -como los medios de comunicación de masas, el currículum de las escuelas, las agendas sociopolíticas intergubernamentales y la investigación académica- a la hora de traducir las ciencias del control en control social a través del espacio pedagógico. De este modo, su investigación ofrece una vía alternativa para que los científicos sociales y los responsables políticos sean conscientes y se responsabilicen de los límites históricos que circunscriben su intervención del cambio social que a menudo se justifica en nombre del progreso y la justicia. Ya ha publicado estudios en libros y en la prestigiosa revista Discourse: Studies in the Politics of Education. ORCID: https://orcid.org/oooo-0003-2298-6432

Catarina S. MarTins is a Professor at the Faculty of Fine Arts, University of Porto. Her current research is related with the historicization of the creative child in education since the end of the i8th century, through governmentality and decolonial perspectives. Recent publications include the following chapters: «Post World War II Psychology, Education, and the Creative Child: Fabricating Differences», «The Fabrication of the Chameleonic Citizen of the Future Through the Rhetoric of Creativity: Governmentality, Competition and Human Capital», and «Arts Education in Portugal in the Eighteenth and Nineteenth Centuries: Drawing and the Governing of the Student». ORCID (oooo-0002-7400-I423). Correo-e: catarina.martins.fbaup@ gmail.com

Ana Luísa Fernandes PAz. Doutorada em Educação. História da Educação, pela Universidade de Lisboa. Professora Auxiliar (Convidada) Instituto de Educação, Universidade de Lisboa. UIDEF, Instituto de Educação, Universidade de Lisboa, Alameda da Universidade, I649-oI3 Lisboa, Portugal. Publicações anteriores: Paz, Ana Luísa: Ser músico em Portugal: Trajetórias do aprender a ser génio, finais do século XIX- 
inícios do século $X X$, Porto, SPCE/De Facto, 2018. Paz, Ana Luísa: «As eternas aprendizes de Euterpe: Mulheres que escrevem sobre música em círculo(s) de sociabilidade (I90I-1930)», Análise Social: Revista do Instituto de Ciências Sociais da Universidade de Lisboa, Lisboa, vol. $53\left(3^{\circ}{ }^{\circ}\right)$, n. ${ }^{\circ} 228$ (2018), pp. 548-570. DOI: http://doi.org/IO.31447/ ASoo032573.2018228.or. Retrieved at: http://analisesocial.ics.ul.pt/documentos/ n228aor.pdf. Paz, Ana Luísa: «As elites musicais em Portugal, finais do século XIX a inícios do século xx: estratégias e padrões de internacionalização», Foro de Educación, Salamanca, I6(25) (2018), pp. I7I-I92. DOI: http://dx.doi.org/Io.I45I6/fde.672. ORCID: https://orcid.org/oooo-0003-4848-8183. Correo-e: apaz@ie.ulisboa.pt

Johanna Sitomaniemi-SAn. Institutional affiliation: Geography Research Unit, University of Oulu, Finland. Research fields: Curriculum studies, history of childhood and education. Recent publications: Sitomaniemi-San, J.: «Suomalainen juurtunut maantiede» [«Finnish rooted geography»], Terra, I3I(4) (2019), pp. 255-257. https://terra. journal.fi/article/view/88707/47871. Sitomaniemi-San, J.: «Tutkivan opettajan rakentuminen akateemisessa opettajankoulutuksessa: Kohti monimutkaisia keskusteluja» [ «The construction of the teacher-as-researcher in academic teacher education: Towards complicated conversations»], en Autio, T.; Hakala, L. y Kujala, T. (eds.): Opetussuunnitelmatutkimus: Keskustelunavauksia suomalaiseen kouluun ja opettajankoulutukseen [Curriculum studies: Discussions on Finnish schooling and teacher education], Tampere, Tampere University Press, 2017, pp. I37-I59. ORCIDhttp://orcid.org/oooo-ooo2-3432-7373. Correo-e: johanna.san@oulu.fi

Daniel Boccacci es docente numerario de Historia y Literatura Italiana de enseñanza secundaria en Italia. En 2014 fue profesor contratado en Sociología de los procesos culturales y de la comunicación y colaboró en los cursos siguientes en la docencia de Sociología de la Educación en la Universidad de Parma, donde es miembro permanente del Centro Interdepartamental de Investigación Social (CIRS). Ha participado como ponente en varios seminarios, entre ellos: La Profesión Docente. Figuras de la vida cotidiana, tramas invisibles (Universidad de Parma, II de octubre de 20II). El pasado 5 de junio de 202I presentó una comunicación en Reinventing Education (Second International Conference) de la prestigiosa revista italiana Scuola Democratica (Universidad de Cagliari, 2-5 de junio de 202I). Ha editado un volumen sobre educación, cultura y tecnología y ha escrito algunos ensayos relacionados con estos ámbitos, como «Il laboratorio Il filo di Arianna. Un'esperienza tra le nuove frontiere dell'apprendimento", La società degli individui, n. 43 (20I2). Actualmente ultima la presentación de su tesis doctoral en Ciencias de la Educación en la Universidad de Granada. ORCID oooo-0002-6509-7865. Correo-e: danielboccacci@libero.it

André Vitor Fernandes dos SANTOS. Graduado em Ciências Biológicas (Licenciatura e Bacharelado) pela Universidade Federal do Rio de Janeiro, é Mestre e Doutor em Educação pela mesma instituição. É Professor Adjunto da Faculdade UnB Planaltina da Universidade de Brasília, atuando na Licenciatura em Ciências Naturais, no Programa de Pós-Graduação em Educação em Ciências (PPGEDUC/UnB) e no Mestrado Profissional em Ensino de Biologia (PROFBIO/UnB). É pesquisador associado ao Grupo de Estudos em História do Currículo, no âmbito do NEC/UFRJ, e ao Grupo de Pesquisa Gerações e Juventude - GERAJU/UnB. Investiga na área de Educação, com ênfase em: Currículo, Políticas Educacionais, Avaliação Educacional e Ensino de Ciências e Biologia. Santos, A. V. F. e Ferreira, M. S.: «Currículo Nacional 
Comum: uma questão de qualidade?», EM ABERTO, v. 33 (2020), pp. 27-44; Ferreira, M. S.; Santos, A. V. F.; Marsico, J.: «Historicizando as reformas curriculares para o ensino e a formação de professores: a inovação curricular em pauta», em Santos, Sandro Prado; Ferreira, Gustavo Lopes e Vigário, Ana Flávia (orgs.). (Bio)grafias: nós e entrenós na educação em ciências e biologia, . . ${ }^{a}$ ed., Uberlândia, Culturatrix, 2021, v. m pp. I69-I90. ORCID: https://orcid.org/oooo-0003-3103-2569. Correo-e: andrevfsantos@ gmail.com

Natalia Pais Álvarez. Doctora en Educación por la Universidad de La Laguna. Profesora de Lengua Española y Literatura en Secundaria. Investigadora del CEILAM y del equipo pedagógico Paideia Hermenéutica de HERCRITIA de la Universidad de La Laguna. Ha indagado en los campos de la educación latinoamericana, el pensamiento transdisciplinar, las aportaciones pedagógicas de la posmodernidad y la reconstrucción de la memoria y el discurso pedagógico desde la literatura contemporánea. También ha elaborado diversos estudios sobre Justicia Social. Ha participado en numerosas publicaciones, artículos y capítulos de libro, congresos, seminarios y jornadas nacionales e internacionales. ORCID iD: oooo-0oo2-7158-764I. Correo-e: natalyapais@gmail.com

María Lourdes GonZALEZ-Luis. Catedrática de Historia de la Educación en la Universidad de La Laguna, ha participado en varios proyectos de investigación financiados por el Ministerio de Ciencia e Innovación de España. Directora de cuatro Expertos sobre Políticas públicas y Gobierno abierto. IP del Erasmus+ TOGIVE, investigadora del Proyecto Erasmus+ Crisálida. Cofundadora del CEILAM (Centro de Estudios Interdisciplinares Latinoamericanos). Directora del Máster de Estudios Pedagógicos Avanzados. Coordinadora de las líneas de investigación histórico-teóricas del Programa de Doctorado en Educación. Coordinadora del grupo de investigación PEDACRI (Pedagogía Crítica) y de la sección Paideia Hermenéutica de la Cátedra Internacional de Hermenéutica Crítica (HERCRITIA) co-liderada por la UNED y la ULL. Son numerosas sus publicaciones, así como su participación en congresos, seminarios y conferencias nacionales e internacionales. ORCID iD: 0000-0003-097I-4758. Correo-e:mlgonzal@ull.edu.es

Pedro Perera Méndez. Doctor en Educación, profesor de la Facultad de Educación de la Universidad de La Laguna, profesor del Máster de Estudios Pedagógicos Avanzados y de los cursos de los expertos universitarios sobre «Gobernabilidad, desarrollo local y equidad» y «Liderazgo político y gobernabilidad». Investigador del CEILAM, PEDACRI y de la Cátedra de Hermenéutica crítica de la UNED-ULL. Investigador y docente en cursos en formato MOOC sobre Gobierno Abierto, Gestión de la información pública y Valores democráticos para el proyecto Erasmus+ «TOGIVE»y el Erasmus+ Crisálida. Ha publicado artículos y capítulos de libros sobre la temática de pedagogía social, construcción de ciudadanías y educación política. Actualmente centrado en investigaciones sobre los populismos y sus consecuencias en la sociedad actual. ORCID iD: oooo-0002-2065-I7II. Correo-e: ppereram@ull.edu.es

Andrés GonzÁlez Novoa. Doctor en Educación, profesor de la Facultad de Educación de la Universidad de La Laguna. Profesor del Máster de Estudios Pedagógicos Avanzados. Investigador del CEILAM, del grupo PEDACRI y del equipo pedagógico de HERCRITIA. Profesor de los expertos universitarios sobre «Democracia y partidos políticos»; "Gobernabilidad, desarrollo local y equidad» y «Liderazgo político 
y gobernabilidad». Codirector de numerosos cursos en formato MOOC sobre Gobierno Abierto, Gestión de la información pública y Valores democráticos para el proyecto Erasmus+ TOGIVE y en formato NOOC sobre Construcción democrática, gobernabilidad, cultura democrática y gestión de políticas públicas para la FGULL. Investigador coordinador del Proyecto Erasmus+ Crisálida. ORCID iD: o00o-00032578-8888. Correo-e: agonzaln@ull.edu.es

Natalia García. Prof. en Ciencias de la Educación, Universidad Nacional de Rosario. Argentina (UNR). Dra. en Educación, Universidad Nacional de Entre Ríos (UNER). Argentina. Prof. Adjunta Historia Socio Política del Sistema Educativo Argentina. Ciencias de la Educación. Facultad de Humanidades y Artes, UNR. Directora del Instituto de Investigaciones "Adolfo Prieto", Facultad de Humanidades y Artes, UNR. Co-directora del Centro de Estudios en Historia de la Educación Reciente Argentina (HEAR) de la Facultad de Humanidades y Artes, UNR. Dos publicaciones recientes: García, N.: «Historia y (des)memorias del icónico libro ¿Maestro pueblo o maestro gendarme? de María Teresa Nidelcoff», Diálogo Educacional, 6I (2019), pp. 55I-575. http://doi.org/I0.7213/I98I-4I6X.19.06I.DSo3. García, N.: «Historiografía y memorias en la educación argentina reciente: balances políticos-académicos y aportes desde un estudio de caso", Perspectivas e Diálogos: Revista de História Social e Práticas de Ensino, 3 (2019), pp. 173-198. https://www.revistas.uneb.br/index.php/nhipe/article/view/6558/4190. ORCID: ttps://orcid.org/oooo-0003-3204-56IX. Correo-e: nataliagr5@gmail.com

Jaime Caiceo Escudero. Doctor en Ciencias de la Educación (Pontificia Universidad Católica Argentina, 1996). Académico de Postgrado de la Universidad de Santiago de Chile y profesor invitado al Doctorado en Educación por la Universidad Nacional de Cuyo en Mendoza, Argentina y la Universidad Católica de Cuyo en San Juan, Argentina. Líneas de investigación: historia de la educación, gestión educacional y filosofía de la ciencia. Publicaciones recientes: Caiceo, J.: «El Desarrollo de la Educación Secundaria durante la primera mitad del siglo Xx en Chile: Liceos Renovados», Revista da FAEEBA-Educação e Contemporaneidade, Salvador, v. 29, n. 59 (jul./set. 2020), pp. I29-I43. DOI: https://doi.org/I0.21879/faeeba2358-0I94.2020.v29.n59.pI29-I43. Caiceo, J.: «Liderazgo y toma de decisiones a nivel de una unidad educativa de Chile», Papeles Salmantinos de Educación, Universidad Pontificia de Salamanca, n. 24 (2020), pp. 93II5. ORCID iD is: https://orcid.org/oooo-00o2-2808-I40X. Correo-e: jcaiceo@hotmail.com;

María Aparecida Corrêa Custódio. Realizou a graduação em Pedagogia pela Faculdade de Educação da USP, o mestrado em Ciências da Religião pela Faculdade de Filosofia e Ciências da Religião da Universidade Metodista de São Paulo, o doutorado em Educação pela Faculdade de Educação da USP. É Profa. Associada da Universidade Federal do Maranhão (campus Imperatriz), atuando no Curso de Pedagogia e no Mestrado em Sociologia. Tem experiência na área de Educação e Ciências da Religião. Trabalha os temas: História da educação e religião, História da educação dos indígenas, Educação e questões étnico-raciais, Políticas educacionais inclusivas, Religião e Gênero. Autora do livro $A$ invenção do cotidiano feminino: formação e trajetória de uma congregação católica (1880-1909), com dezenas de artigos publicados em periódicos, livros e anais de congresso. ORCID: 0000-0003-2962-2228. Correo-e: mac. custodio@ufma.br 
Isabel Camilo de Camargo. Pós-doutoranda do Programa de Pós-Graduação em Educação da Universidade Federal de Mato Grosso - UFMT, campus de Rondonópolis, bolsista Capes. Principais temas de atuação: Pesquisa em História, Pesquisa em Educação, Prática de Ensino em História, História do Brasil Imperial, História de Mato Grosso do Sul. Publicações recentes: Camargo, Isabel Camilo de e Gomes, Aguinaldo Rodrigues: "A escravidão em Sant'Ana de Paranaíba/MT no século xix: debates historiográficos e a mulher escravizada», Sankofa (São Paulo), v. I3 (2020), pp. 75-94. Camargo, Isabel Camilo de: «As relações étnico-raciais na sociedade brasileira: suas implicações na Educação e nas Legislações Educacionais», REIDICS, v. 6 (2020), pp. 6I-76. ORCID:https://orcid.org/oooo-oooI-7066-62IX. Correo-e: isabelc_camargo@ hotmail.com

Aguinaldo Rodrigues Gomes. Docente do curso de História da Universidade Federal de Mato Grosso do Sul - UFMS, campus de Aquidauana; do Programa de Pós-Graduação em Estudos Culturais/UFMS e do Programa de Pós-Graduação em Educação da Universidade Federal de Mato Grosso - UFMT, campus de Rondonópolis. Principais temas de atuação: História e Ditadura, Educação e Comunismo, Gênero e Sexualidade, Estudos Culturais. Publicações recentes: Camargo, Isabel Camilo de e Gomes, Aguinaldo Rodrigues: "A escravidão em Sant'Ana de Paranaíba/MT no século XIX: debates historiográficos e a mulher escravizada», Sankofa (São Paulo), v. I3 (2020), pp. 75-94. Gomes, Aguinaldo Rodrigues; Silva, Robson Pereira da e De Lion, Antonio Ricardo Calori: «Educação \& emancipação pela agência dos movimentos sociais de sexualidade e de gênero notas para uma pedagogia da diferença», História Revista, v. 25 (2020), pp. 06-36. ORCID: https://orcid.org/oooo-0oo2-2398-8088. Correo-e: aguinaldorod@gmail.com

Xosé Manuel Souto GonzÁlez es catedrático de Didáctica de las ciencias sociales en la Universitat de València. Coordinador del proyecto Gea-Clio desde 1989, que ha propuesto una alternativa a la organización académica de la enseñanza de ciencias sociales y geografía e historia en Primaria y Secundaria. Coordinador desde 2008 del Geoforo Iberoamericano de Educación, Geografía y Sociedad, que abarca a numerosas universidades de países como Argentina, Brasil, Colombia, Costa Rica, Chile, México, Perú, Portugal, Venezuela y España. Las últimas publicaciones son: Campos Pais, Benito; Fita Esteve, Sara; Martínez Camarena, María y Souto González, Xosé Manuel: «La pandemia y el Geoforo Iberoamericano en 2020», Ar@cne. Revista Electrónica de Recursos de Internet sobre Geografía y Ciencias Sociales, Barcelona, Universidad de Barcelona, I de abril de 202I, vol. XXV, n. ${ }^{\circ}$ 253. DOI: https://doi.org/Io.I344/ara202I.253.34376; Souto González, Xosé Manuel y Sobrino López, Diego: «Sostenibilidad y transformación ambiental», Iber: Didáctica de las Ciencias Sociales, Geografía e Historia, n. ${ }^{\circ}$ IO2 (202I), pp. 4-7, ISSN iı33-98io,; Crespo Castellanos, José Manuel; Sebastià Alcaraz, Rafael y Souto González, Xosé M.: «La enseñanza de la Geografía en el Máster de Formación del Profesorado», en Gómez Carrasco, Cosme Jesús; Souto, Xosé Manuel y Miralles Martínez, Pedro (eds.): Enseñanza de las Ciencias Sociales para una ciudadanía democrática. Estudios en homenaje del profesor Ramón López Facal, Barcelona, Octaedro, 202I, pp. 55-70), ISBN: 978-84-I86I5-9I-7 D.L. B 8633-202I; Parra Monserrat, David; Souto González, Xosé Manuel y Palacios Mena, Nancy: «Chapter 5 The representation of geographical and historical education among teachersin- training: An Ibero-American perspective», en Gómez Carrasco, Cosme Jesús; Miralles Martínez, Pedro y López Facal, Ramón: Handbook of Research on Teacher 
Education in History and Geography, Berlin, Peter Lang, 2021, pp. II5-136, ISBN 978-3-63I-8I897-8 (Print); E-ISBN 978-3-63I-84838-8 (E-PDF); DOI: $10.3726 /$ bi809I ; Campo-Pais, B.; Morales-Hernández, A. J.: Morote-Seguido, Á. y Souto-González, X. M.: «Environmental problems and Geographic education. A case study: Learning about the climate and landscape in Ontinyent (Spain)», Humanit Soc Sci Commun, 8 (90) (202I). https://doi.org/I0.I057/s4I599-02I-0076I-6 (QI SJR-Palgrave). Correo-e: xose.manuel.souto@uv.es

Francisco F. García Pérez. Actualmente jubilado, ha sido catedrático de Didáctica de las Ciencias Sociales en la Facultad de Ciencias de la Educación de la Universidad de Sevilla; anteriormente fue profesor de Geografía e Historia en el IES Martínez Montañés, de Sevilla. Es licenciado en Historia General por la Universidad de Sevilla y doctor en Pedagogía por dicha Universidad. Forma parte del colectivo Fedicaria (http://www. fedicaria.org/) y de la Red IRES (https://redires.net/). Ha participado en investigaciones sobre innovaciones educativas y sobre formación del profesorado, especialmente en relación con la educación para la ciudadanía. Es autor de diversos libros, capítulos de libros y artículos en revistas relacionadas con la educación. Dos publicaciones recientes: García Pérez, F. F.; Burgos Sánchez, M. y Guerrero Fernández, A.: «La construcción de la ciudadanía en la formación inicial del profesorado de Educación Infantil», Didáctica de las Ciencias Experimentales y Sociales, 39 (2020), pp. 47-64. DOI: 10.7203/DCES.39.I7I2I. https://ojs.uv.es/index.php/dces/article/view/I7I2I/pdf. García Pérez, F. F.: «Algunas dificultades del profesorado de Geografía para educar en ciudadanía», Didacticae, 9 (202I), pp. 39-52. https://revistes.ub.edu/index.php/didacticae/article/view/325IO. DOI: https://doi.org/IO.I344/did.202I.9.39-52 ORCID: http:// orcid.org/oooo-0002-9988-3231. Correo-e: ffgarcia@us.es 
